\title{
Multisite pain and self-reported falls in older people: systematic review and meta- analysis
}

Victoria K. Welsh ${ }^{1 *}$, Lorna E. Clarson ${ }^{1}$, Christian D. Mallen ${ }^{1}$ and John McBeth ${ }^{2}$

\begin{abstract}
Background: Multisite pain and falls are common in older people, and isolated studies have identified multisite pain as a potential falls risk factor. This study aims to synthesise published literature to further explore the relationship between multisite pain and falls and to quantify associated risks.

Methods: Bibliographic databases were searched from inception to December 2017. Studies of community-dwelling adults aged 50 years and older with a multisite pain measurement and a falls outcome were included. Two reviewers screened articles, undertook quality assessment and extracted data. Random-effects meta-analysis was used to pool the effect estimate (odds ratio (OR) and 95\% confidence interval (95\%Cl)). Heterogeneity was assessed by $P^{2}$; sensitivity analyses used adjusted risk estimates and exclusively longitudinal studies.

Results: The search identified 49,577 articles, 3145 underwent abstract review, 22 articles were included in the systematic review and 18 were included in the meta-analysis. The unadjusted pooled OR of $1.82(95 \% \mathrm{Cl} 1.55-2.13)$, demonstrating that those reporting multisite pain are at increased risk of falls, is supported by the adjusted pooled OR of 1.56 (95\%Cl 1.39-1.74). Multisite pain predicts future falls risk ( $\mathrm{OR}=1.74(95 \% \mathrm{Cl} 1.57-1.93))$. For high-quality studies, those reporting multisite pain have double the odds of a future fall compared to their pain-free counterparts.

Conclusion: Multisite pain is associated with an increased future falls risk in community-dwelling older people. Increasing public awareness of multisite pain as a falls risk factor and advising health and social care professionals to identify older people with multisite pain to signpost accordingly will enable timely falls prevention strategies to be implemented.
\end{abstract}

Keywords: Musculoskeletal pain, Falls prevention, Public health

\section{Introduction}

Falls are common in older people, and prevalence increases with advancing age, from $20.8 \%$ in adults aged 60-69 years to $33.2 \%$ for those aged 80 years and older [1]. Known falls risk factors include a history of previous fall, women in the oldest old age groups, particular medication use (for example, benzodiazepines, psychotropics, diuretics and sedatives) and polypharmacy [2]. Specific co-morbidities (including circulatory disease, chronic obstructive pulmonary disease, arthritis) and rising chronic disease burden increase falls risk, along with

\footnotetext{
* Correspondence: v.welsh@keele.ac.uk

${ }^{1}$ Arthritis Research UK Primary Care Centre, Research Institute for Primary Care \& Health Sciences, Keele University, Keele, Staffordshire ST5 5BG, UK
} Full list of author information is available at the end of the article reduced physical functioning, impaired cognition and visual impairment [2].

Falls are associated with poor outcomes; they are responsible for 65,000 hip fractures annually in the UK [3], cost the National Health Service $£ 2.3$ billion per year [4] and can lead to increased need for social support which impacts upon families, communities and employers.

Given the prevalence of falls and their potentially devastating consequences, falls prevention is essential to improving health and well-being of older people. Falls prevention guidelines are based upon management of known risk factors and are widely implemented by health care professionals, yet falls remain a common component of ageing. Multisite pain, defined as pain in more than one part of the body, has been proposed as a

(c) The Author(s). 2019 Open Access This article is distributed under the terms of the Creative Commons Attribution 4.0 International License (http://creativecommons.org/licenses/by/4.0/), which permits unrestricted use, distribution, and 
novel risk factor for falls [5]. A number of more recent studies have examined this [6, 7]; however, the subject remains under-investigated with comparatively small cohorts and consideration of limited putative confounders of the multisite pain and falls relationship.

As the first study to draw together published evidence, this research tests the hypothesis that multisite pain increases the risk of falls in older people, it seeks to establish the nature of the relationship between multisite pain and falls amongst different community-dwelling populations and to quantify this risk using a systematic review and meta-analysis.

\section{Methods}

The systematic review followed the Preferred Reporting Items for Systematic Reviews and Meta-Analyses (PRISMA) guidelines.

\section{Search strategy and selection criteria}

Seventeen online bibliographic databases were searched from inception until 7 December 2017. The full list of information sources can be found in Additional file 1 and included MEDLINE, Embase, The Cochrane Library, Cumulative Index to Nursing and Allied Health Literature (CINAHL), the British Nursing Index, PsychInfo, Conference Proceedings Citation Index and charity or society websites including AgeUK and the British Geriatric Society. Reference lists of relevant publications were searched, and authors were contacted to obtain further information and to identify additional studies.

Multisite pain was searched using the term 'pain' as either an exploded Medical Subject Headings (MeSH) term or as free text; other terms used to capture multisite pain (musculoskeletal diseases, osteoarthritis, arthralgia, pain(ful) hip, pain(ful) knee, pain(ful) ankle, pain(ful) foot) were exploded MeSH terms and searched as free text. All the pain-related terms were combined using the 'OR' operator. Fall-related MeSH varied across databases and comprised 'accidental falls', 'falls risk,' 'falls risk assessment' and 'falling'; each of these terms were exploded. The free-text term 'fall"' was used to cover fall, falls, falling, fallen and faller. Each of the fall-related search outputs were combined using the 'OR' operator. All of the pain-related terms and all of the fall-related terms were then combined using the 'AND' operator. The pooled results were limited to those that contained both pain-related and fall-related search terms in either the title, abstract or associated key words.

Searches were limited to human studies only; no other limitations were applied. Studies were excluded if the study population resided in nursing homes or were hospital inpatients or if the pain measure did not quantify the number of pain sites. Authors of studies appearing to have collected information on the number of pain sites or falls but not including this information in the published research were contacted. Studies were excluded when no further information was available.

Articles were included if the population comprised community-dwelling adults aged 50 years and older, a measure of multisite pain was present, the study included a no-pain group and information on falls was available.

Titles and abstracts were screened by two reviewers (VKW, LEC). Methodological quality was assessed by two reviewers independently (VKW, LEC) and a consensus recorded. The Quality in Prognosis Studies (QUIPS) tool, a validated and widely adopted measure used to assess bias in prognostic studies [8], was used to critically appraise the 22 included studies.

This tool was used to assess included studies since key elements of assessing bias in cross-sectional studies are included within this tool and using the same tool for all identified articles enabled comparison between studies.

\section{Data extraction and analysis}

Data extraction from selected studies was undertaken by two reviewers (VKW, LEC) using a study-specific proforma; information was stored in a purpose-designed spreadsheet. Information extracted included country of study setting, type of study, sample size, participant characteristics, recruitment details, inclusion and exclusion criteria, follow-up duration (where relevant), response rate, loss to follow-up, description and classification of multisite pain, potential confounders, falls definition, falls measurement, fall-related outcomes including effect estimates, study conclusion and funding source.

Where possible, data for both unadjusted analysis and the most highly adjusted analysis were extracted from each study. Effect estimates were standardised to odds ratios where possible.

All identified articles were included in the systematic review and examined using narrative synthesis. Random-effects meta-analysis was performed to produce unadjusted and adjusted summary effect estimates (odds ratios (OR) with 95\% confidence intervals (CI)). Heterogeneity across studies was measured using $I^{2}$ statistic and Cochran's $Q$ test. Publication bias was assessed using funnel plots. For analyses including ten or more studies, Begg's and Eggar's tests were used to examine funnel plot symmetry and publication bias. Sensitivity analyses tested the multisite pain-falls relationship in (i) studies that presented adjusted analyses, (ii) longitudinal studies, and (iii) studies considered at low risk of bias. Stata Statistical Software Release 14 was used.

\section{Results}

\section{Search results}

The search yielded 49,577 titles, of which 3145 abstracts were screened, 478 full texts were read, 22 studies were 
included in the systematic review (representing 40,705 participants) and 18 were included in the meta-analysis; the study flowchart is presented in Fig. 1. Two studies included the same study population, one as a cross-sectional study [9] and one a prospective cohort study [10]; both are included in the narrative review.

\section{Study characteristics}

Additional file 2 presents included studies' characteristics. A total of 24,935 participants were included from eight countries. Thirty-two percent of studies were from North America, 27\% from Japan, 18\% from Australia and New Zealand, 14\% from the UK and 14\% from the rest of the world. A cross-sectional study design was used for $50 \%$ of studies, $41 \%$ utilised a prospective cohort design and $9 \%$ were case-control studies.

\section{Pain and falls measures}

Pain was defined using different time frames. For example, pain presence ranged from simply the presence

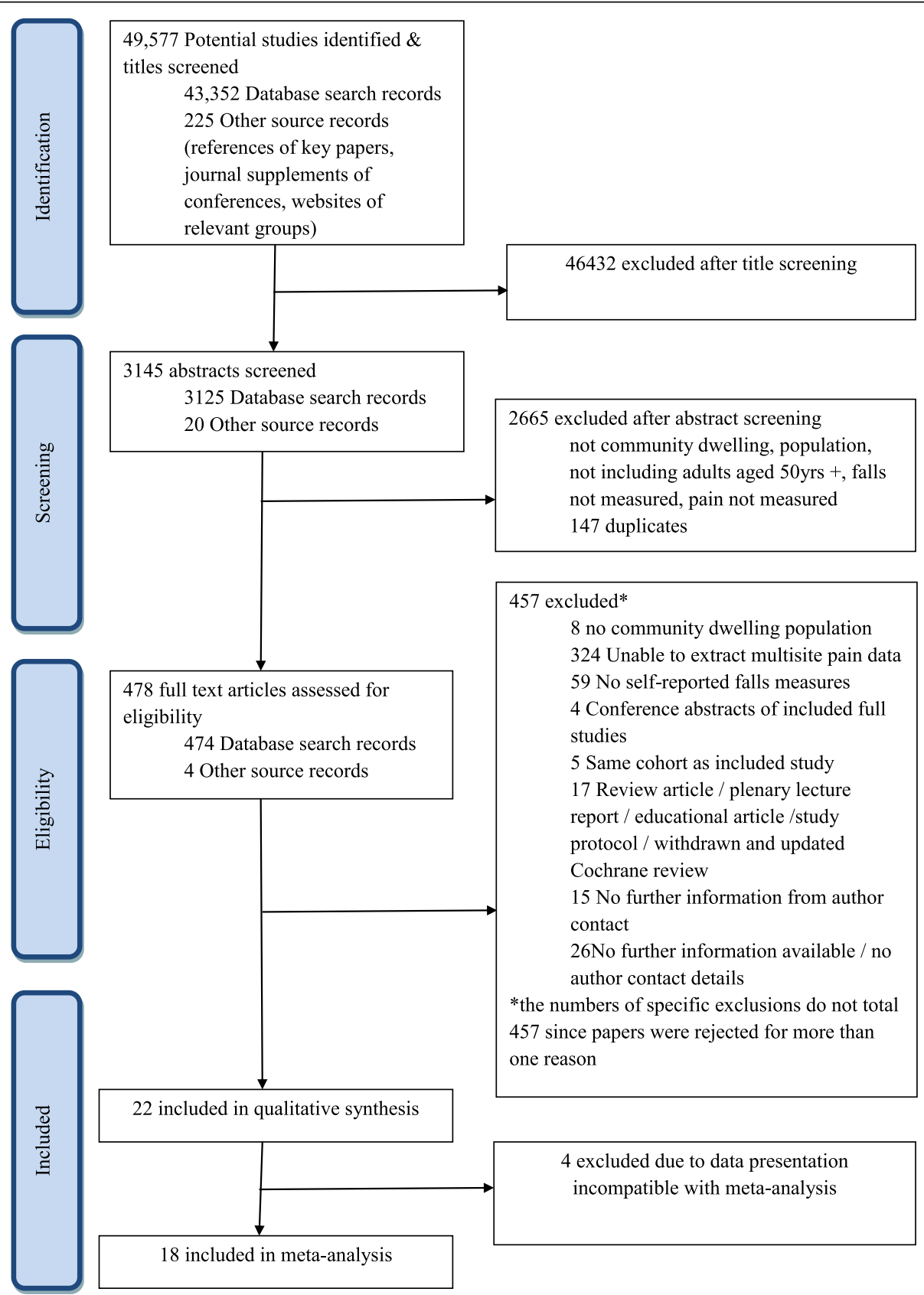

Fig. 1 Study flowchart detailing the screening and selection process 
of pain' to 'pain experienced in the last week' through 'pain in the last 12 months' and 'pain experienced for at least 3 months in the previous year'. The requirement of current pain alongside a history of pain in the preceding year was used by $14 \%$ of studies. Twenty-seven percent of studies used varying definitions to elicit longer-lasting pain rather than acute episodes, for example, 'persistent pain', pain on 'most days,' 'pain on most days for at least a month', 'pain lasting for 1 month or more', or 'pain lasting for at least 3 months'.

Severity of pain was included by $9 \%$ of studies as part of their classification, and one study used pain associated with radiographic changes to ascertain the pain population.

Multisite pain was defined differently across studies; $41 \%$ of studies used body manikins or questionnaires to take account of pain in multiple different body sites' status, 32\% explored pain in specific body parts (for example, upper, middle or lower back and presence or absence of hip pain; or low back pain and hip pain); $27 \%$ used presence and number of tender joints as a measure of pain caused by inflammatory conditions and $9 \%$ measured number of tender points in fibromyalgia.

The gold standard falls definition provided by the Prevention of Falls Network Europe as 'an unexpected event in which the participants come to rest on the ground, floor, or lower level' [11] was used by $32 \%$ of studies; $45 \%$ did not explicitly state a definition for falls, and the remaining studies provided definitions that were adapted from the gold standard definition.

Fifty-five percent of studies collected retrospective information on falls in the preceding 12 months, $18 \%$ collected retrospective information on falls in the preceding 6 months, $23 \%$ studies collected prospective information on falls for the 12 months following baseline and 5\% collected information on falls for 18 months following the baseline survey. The gold standard method of prospective falls data (use of a falls calendar) was used by $14 \%$ of studies.

\section{Quality assessment}

Additional file 3 provides a summary of the risk of bias assessments. Overall, $41 \%$ of studies were deemed high risk of bias [12-20], $41 \%$ were medium risk [7, 9, 10, $21-26]$ and $18 \%$ were considered to be at a low risk of bias $[5,6,27,28]$. The most common reasons papers were considered at high risk of bias were unclear description of study participation and study attrition such that bias limitation was unable to be determined. Descriptions of pain measures, falls measures and confounding measures were also limited such that bias potential was deemed partially limited.

\section{Pain and falls relationship}

Additional file 4 presents individual study results, including effect estimates and confounders that were included in calculations. Eighty-two percent of studies demonstrated a statistically significant association between the presence of multisite pain and falls when adjusting for confounding factors [5-7, 9, 10, 12, 13, 15, $16,18-23,26,28] ; 18 \%$ of studies found no statistically significant relationship between multisite pain and falls after adjusting for confounders [14, 17, 24, 27]. Studies that classified multisite pain by number of pain sites or included a measure of widespreadness found a linear correlation between the number of pain sites and an increasing risk of falls $[5,6,22,23,25]$.

\section{Cross-sectional relationship}

Eighty-five percent of studies demonstrated a statistically significant cross-sectional relationship between the presence of multisite pain and previous history of falls when adjusting for confounding factors $[6,7,9,12,13,15,16,18-21] ; 15 \%$ of studies found no statistically significant cross-sectional relationship after adjusting for confounders [14, 17].

\section{Longitudinal relationship}

Follow-up periods ranged from 12 months [10, 22, 2528] through 18 months [5] to 3 years [23, 24]. The retrospective recall periods of falls in the follow-up period ranged from monthly for 12 months [10, 27], four-monthly for 12 months [25, 26], six-monthly for 3 years [23] to the 12 months prior to follow-up [22, 24]. Two studies used daily falls calendars to record falls $[5,28]$. All prospective studies reported a trend towards multisite pain increasing the risk of self-reported future fall $[5,10,22-28] ; 22 \%$ of those did not reach statistical significance [24, 27].

\section{Meta-analysis}

As Fig. 2 demonstrates, the pooled estimate summary for the unadjusted association between multisite pain and falls is an OR $1.82(1.55-2.13)$. Heterogeneity is likely to have significantly affected the pooled estimate with an $I^{2}$ of $68.8 \%$ and a Cochran $Q$ probability of < 0.01 . Additional file 5 demonstrates an asymmetrical funnel plot and Eggar's test statistics $p<0.01$ indicating potential publication bias; Eggar's test $p=0.22$ indicates potential instability of the test when a small number of studies are included. Those studies presenting only mean tender joint counts or where odds ratios are not able to be calculated are not included in the pooled estimate. Ho et al. [16] had bilateral wrist pain included in the unadjusted analysis, and chronicity multisite measure was used from Kitayuguchi et al. [27].

The relationship between multisite pain and falls persisted in sensitivity analyses. Analysis using risk estimates that have been adjusted to be most representative of real-life clinical scenarios (ten studies, $n=14,176$ ) found a summary effect estimate of OR 1.56 (1.39-1.74), 


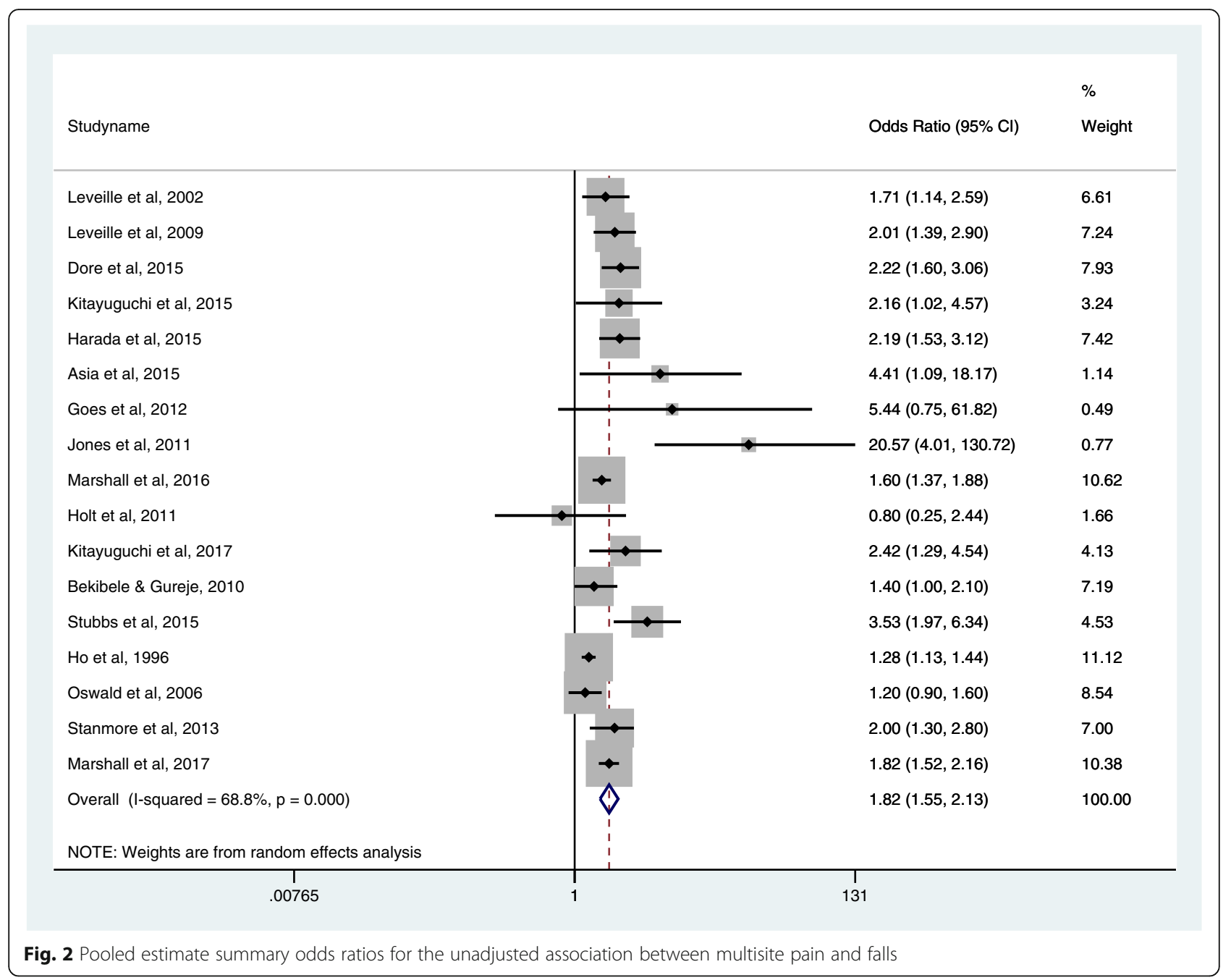

$I^{2}=0$, a symmetrical funnel plot, Begg's test $p=0.09$ and Egger's test $p=<0.01$.

\section{Longitudinal relationship}

Analysis including only longitudinal studies (five studies, $n=14,633)$ found an unadjusted summary risk estimate of 1.74 (1.57-1.93), with an $I^{2}=0.0 \%$, and a symmetrical funnel plot. Analysis including only prospective cohort studies with adjusted risk estimates to represent real-life scenarios (two studies $[23,28], n=1475$ ) found a summary risk estimate of $1.63(1.28-2.07), I^{2}=0.0 \%$ and a symmetrical funnel plot. Analysis including studies considered at low risk of bias (two studies [5, 28], $n=1284$ ) found an unadjusted summary risk estimate of 2.01 (1.54-2.61), $I^{2}=0.0 \%$ and a symmetrical funnel plot.

\section{Discussion}

\section{Summary of findings}

This systematic review and meta-analysis has found that multisite pain is associated with an increased risk of self-reported falls. Analyses adjusted for confounding factors found that those with multisite pain had increased odds of a self-reported fall of 1.56 (1.39-1.74) compared to those with no pain. When considering only those studies with low risk of bias, the odds of sustaining a future fall for those with multisite pain rose to almost double the odds of those falling with no multisite pain.

\section{Differences between studies Study designs}

Cross-sectional, cohort and case-control studies were included in the review. Whilst prospective cohort studies can establish a temporal relationship, the majority of the studies were cross-sectional, a design that precludes causality. All study designs were included in the meta-analysis to maximise study population and thus provide a more precise summary effect estimate of the multisite pain and falls relationship. Sensitivity analysis using only prospective cohort studies confirmed the relationship between the presence of multisite pain and self-reported falls. 


\section{Definition of multisite pain}

Heterogeneity exists between studies over definitions of multisite pain. Definitions ranged from inclusion of many possible different pain sites using a body manikin to indicate pain sites, to studies specifically enquiring about two sites of pain (for example, low back pain and knee pain). It is therefore possible that respondents reporting no pain in the specified locations are misclassified in the no-pain comparator group when pain might be present, but not in the pre-specified locations. This misclassification may suggest that certain pain phenotypes, for example, knee pain and low back pain, contribute a relatively greater falls risk than other pain phenotypes. This requires further exploration to determine which aspect of multisite pain (for example, the number of pain sites per se, or the pattern of pain experienced) is contributing to future falls risk to enable future targeted research and interventions to reduce falls in older people.

\section{Falls outcome measure}

The data collection method for self-reported falls ranged from the gold standard of contemporaneously completing daily falls calendars ( $18 \%$ studies) to recall of previous falls (68\% studies). Relying on recall of falls may introduce misclassification of fallers as non-fallers and thus lead to an underestimation of falls risk, as demonstrated by Hannan et al. who found that people aged 70 years and older were able to recall only $70 \%$ of all falls that had occurred in the previous 3-month period and, of those who fell, $25 \%$ were subsequently misclassified as non-fallers [29].

\section{Strengths and limitations}

This systematic review and meta-analysis is comprehensive, drawing on evidence from multiple sources, including research from around the world and including non-English publications.

This study did not explore the association between multisite pain and falls in residential and care home settings. Although the study was designed to exclude such populations, the search strategy did include residential or nursing home residents and no studies were found that explored the relationship between pain and falls within these populations, thus identifying a future research need.

Seventy-eight percent of studies were considered to have a medium or high risk of bias; the most common potential source of bias was due to omitted reporting of response rates and attrition rates with no accompanying explanations for non-response or drop-outs. If the non-response or drop-outs were due to advancing age, poorer health and increased frailty, then the study may underestimate the risk of falls since those more likely to fall have not been included in the study.
The systematic search found only one study exploring the relationship between multisite pain and injurious falls. Welmer et al. found, in a cohort followed over 10 years, that the hazard ratio for pain in two or more sites and future injurious fall (defined by hospitalisation or receipt of outpatient care because of a fall) was 1.79 (1.192.69) in their adjusted analysis [30]. The link between multisite pain and subsequent injury or secondary health care requirement has not yet been confirmed in the literature and the association between multisite pain and falls requiring primary health care remains unknown. Further large prospective studies are therefore required to confirm the role of multisite pain as a risk factor for future self-reported falls and to explore the association between multisite pain and falls requiring primary and secondary care utilisation to enable economic analyses to be undertaken.

\section{Implications for research and clinical practice}

This meta-analysis confirms those early study findings that multisite pain is associated with an increased risk of future self-reported falls and there are clear biological pathways to explain this link. For example, pain is associated with mobility limitation which in turn leads to more sedentary behaviour, loss of muscle power and thus an increased risk of falls [2]. Further work must now be undertaken to ascertain the relationship between multisite pain and future falls requiring primary and secondary health care use to determine the level of healthcare use associated with such falls and enable health service planning and organisation to help meet the needs of older people at risk of falls on a population level.

\section{Conclusions}

This systematic review and meta-analysis has found that multisite pain is associated with an increased risk of future self-reported fall. Older people with multisite pain must therefore be considered at increased risk of falls. This is an important public health message to disseminate to older people who can self-identify with multisite pain and seek further guidance from health care professionals to reduce their risk of falls. Health and social care professionals who are regularly reviewing older people are advised to identify those with multisite pain and signpost accordingly so that falls prevention management strategies can be implemented in line with current guidelines.

\section{Additional files}

Additional file 1: Systematic review and meta-analysis: databases and data sources searched. (docx $20 \mathrm{~kb}$ )

Additional file 2: Study characteristics for the studies included in the systematic review. (docx 31 kb) 
Additional file 3: Summary of the risk of bias assessments using the Quality in Prognostic Studies tool. (docx 23 kb)

Additional file 4: Multisite pain and falls: individual study results. (docx $26 \mathrm{~kb}$ )

Additional file 5: Funnel plot of publications examining the unadjusted relationship between multisite pain and falls for cross-sectional, cohort and case-controlled studies. (docx $22 \mathrm{~kb}$ )

\section{Abbreviations}

Cl: Confidence interval; CINAHL: Cumulative Index to Nursing and Allied Health Literature; MeSH: Medical Subject Headings; OR: Odds ratio; PRISMA: Preferred Reporting Items for Systematic Reviews and MetaAnalyses; QUIPS: Quality in Prognosis Studies

\section{Acknowledgements}

The authors gratefully acknowledge contributions from Dr Charles Bekibele, Dr Oye Gureje and Dr Kelly Holt for providing additional analyses. The authors are grateful for the assistance from the Systematic Review team at the Arthritis Research UK Primary Care Centre, Keele University for their support with search strategy development and implementation.

\section{Funding}

The work was supported by the National Institute of Health Research (NIHR) (Doctoral Research Fellowship 2011-04-147) to VKW. The views expressed are those of the authors and not necessarily those of the National Health Service, the NIHR or the Department of Health.

\section{Availability of data and materials}

All data is available in the original references.

\section{Authors' contributions}

VKW conceived and designed the study, screened the titles, abstracts and full texts, undertook quality appraisal and data extraction, performed the analysis and prepared the manuscript. LC participated in title, abstract and full-text screening, quality appraisal and read and approved the final manuscript. CDM and JM conceived the study and read and approved the final manuscript. All authors read and approved the final manuscript.

\section{Ethics approval and consent to participate}

not applicable.

\section{Consent for publication}

not applicable

\section{Competing interests}

The authors declare that they have no competing interests.

\section{Publisher's Note}

Springer Nature remains neutral with regard to jurisdictional claims in published maps and institutional affiliations.

\section{Author details}

${ }^{1}$ Arthritis Research UK Primary Care Centre, Research Institute for Primary Care \& Health Sciences, Keele University, Keele, Staffordshire ST5 5BG, UK. ${ }^{2}$ Arthritis Research UK Centre for Epidemiology, Faculty of Biology, Medicine and Health, The University of Manchester, Manchester M13 9PT, UK.

Received: 28 August 2018 Accepted: 6 February 2019

Published online: 22 February 2019

\section{References}

1. Gale CR, Cooper C, Aihie Sayer A. Prevalence and risk factors for falls in older men and women: the English longitudinal study of ageing. Age Ageing. 2016;45(6):789-94.

2. Todd C, Skelton D. What are the main risk factors for falls among older people and what are the most effective interventions to prevent these falls? Copenhagen: WHO Regional Office for Europe (Health Evidence Network report); 2004. Available from http://www.euro.who.int/document/E82552. pdf. Accessed 16th November 2016.
3. Royal College of Physicians. National Hip Fracture Database (DHFD) annual report 2015. London: Royal College of Physicians; 2015.

4. National Institute for Health and Care Excellence. Falls in older people: National Institute for Health and Clinical Excellence; 2017. Available from https://www.nice.org.uk/guidance/qs86. Accessed 20 May 2017

5. Leveille SG, Jones RN, Kiely DK, Hausdorff JM, Shmerling RH, Guralnik JM, et al. Chronic musculoskeletal pain and the occurrence of falls in an older population. JAMA. 2009;302(20):2214-21.

6. Patel KV, Phelan EA, Leveille SG, Lamb SE, Missikpode C, Wallace RB, Guralnik JM, Turk DC. High prevalence of falls, fear of falling, and impaired balance in older adults with pain in the United States: findings from the 2011 National Health and Aging Trends Study. J Am Geriatr Soc. 2014; 62(10):1844-52.

7. Stubbs B, Eggermont L, Patchay S, Schofield P. Older adults with chronic musculoskeletal pain are at increased risk of recurrent falls and the brief pain inventory could help identify those most at risk. Geriatr Gerontol Int. 2015;15(7):881.

8. Hayden JA, Cote P, Bombardier C. Evaluation of the quality of prognosis studies in systematic reviews. Ann Intern Med. 2006;144(6):427-37.

9. Brenton-Rule A, Dalbeth N, Menz HB, Bassett S, Rome K. Foot and ankle characteristics associated with falls in adults with established rheumatoid arthritis: a cross-sectional study. BMC Musculoskelet Disord. 2016;17:22.

10. Brenton-Rule A, Dalbeth N, Menz HB, Bassett S, Rome K. Are foot and ankle characteristics associated with falls in people with rheumatoid arthritis? A prospective study. Arthritis Care Res (Hoboken). 2017;69(8):1150-5.

11. Lamb SE, Jorstad-Stein EC, Hauer K, Becker C, Prevention of Falls Network Europe and Outcomes Consensus Group. Development of a common outcome data set for fall injury prevention trials: the Prevention of Falls Network Europe consensus. J Am Geriatr Soc. 2005;53(9):1618-22.

12. Asai T, Misu S, Sawa R, Doi T, Yamada M. Multi-chronic musculoskeletal pain is a useful clinical index to predict the risk of falls in older adults with normal motor function. Aging Clin Exp Res. 2015;27:711-6.

13. Furuya T, Yamagiwa K, Ikai T, Inoue E, Taniguchi A, Momohara S, Yamanaka $H$. Associated factors for falls and fear of falling in Japanese patients with rheumatoid arthritis. Clin Rheumatol. 2009;28(11):1325-30.

14. Góes M, Leite N, Shay L, Homann D, Stefanello MF, Rodacki LF. Functional capacity, muscle strength and falls in women with fibromyalgia. Clin Biomech (Bristol, Avon). 2012;27(6):578-84.

15. Harada K, Shibata A, Oka K, Nakamura Y. Association of musclestrengthening activity with knee and low back pain, falls, and health-related quality of life among Japanese older adults: a cross-sectional survey. J Aging Physical Act. 2015;23(1):1-8.

16. Ho SC, Woo J, Chan SS, Yuen YK, Sham A. Risk factors for falls in the Chinese elderly population. J Gerontol A Biol Sci Med Sci. 1996;51(5):M195-8.

17. Holt KR, Noone PL, Short K, Elley CR, Haavik H. Fall risk profile and quality-of-life status of older chiropractic patients. J Manip Physiol Ther. 2011;34(2):78-87.

18. Jones KD, King LA, Mist SD, Bennett RM, Horak FB. Postural control deficits in people with fibromyalgia: a pilot study. Arthritis Res Ther. 2011;13(4):R127.

19. Kitayuguchi J, Kamada M, Okada S, Kamioka H, Mutoh Y. Association between musculoskeletal pain and trips or falls in rural Japanese community-dwelling older adults: a cross-sectional study. Geriatr Gerontol Int. 2015;15(1):54-64.

20. Oswald AE, Pye SR, O'Neill TW, Bunn D, Gaffney K, Marshall T, Silman A, Symmons DP. Prevalence and associated factors for falls in women with established inflammatory polyarthritis. J Rheumatol. 2006;33(4):690-4.

21. Hayashibara M, Hagino H, Katagiri H, Okano T, Okada J, Teshima R. Incidence and risk factors of falling in ambulatory patients with rheumatoid arthritis: a prospective 1-year study. Osteoporos Int. 2010;21(11):1825-33.

22. Stanmore EK, Oldham J, Skelton DA, O'Neill T, Pilling M, Campbell AJ, Todd C. Risk factors for falls in adults with rheumatoid arthritis: a prospective study. Arthritis Care Res (Hoboken). 2013;65(8):1251-8.

23. Bekibele $\mathrm{CO}$, Gureje O. Fall incidence in a population of elderly persons in Nigeria. Gerontology. 2010;56(3):278-83.

24. Doré AL, Golightly YM, Mercer VS, Shi XA, Renner JB, Jordan JM, Nelson AE. Lower-extremity osteoarthritis and the risk of falls in a community-based longitudinal study of adults with and without osteoarthritis. Arthritis Care Res (Hoboken). 2015;67(5):633-9.

25. Leveille SG, Bean J, Bandeen-Roche K, Jones R, Hochberg M, Guralnik JM. Musculoskeletal pain and risk for falls in older disabled women living in the community. J Am Geriatr Soc. 2002;50(4):671-8.

26. Marshall LM, Litwack-Harrison S, Makris UE, Kado DM, Cawthon PM, Deyo RA, Carlson HL, Nevitt MC, Osteoporotic Fractures in Men Study (MrOS) Research group. A prospective study of back pain and risk of falls among 
older community-dwelling men. J Gerontol A Biol Sci Med Sci. 2017;72(9): 1264-9.

27. Kitayuguchi J, Kamada M, Inoue S, Kamioka H, Abe T, Okada S, Mutoh Y. Association of low back and knee pain with falls in Japanese communitydwelling older adults: a 3-year prospective cohort study. Geriatr Gerontol Int. 2017;17(6):875-84.

28. Marshall LM, Litwack-Harrison S, Cawthon PM, Kado DM, Deyo RA, Makris UE, Carlson HL, Nevitt MC; for the Osteoporotic Fractures (SOF) Research Group. A prospective study of back pain and risk of falls among older community-dwelling women. J Gerontol A Biol Sci Med Sci 2016;71(9): 1177-1183.

29. Hannan MT, Gagnon MM, Aneja J, Jones RN, Cupples LA, Lipsitz LA, Samelson EJ, Leveille SG, Kiel DP. Optimizing the tracking of falls in studies of older participants: comparison of quarterly telephone recall with monthly falls calendars in the MOBILIZE Boston study. Am J Epidemiol. 2010;171(9): 1031-6.

30. Welmer AK, Rizzuto D, Calderón-Larrañaga A, Johnell K. Sex differences in the association between pain and injurious falls in older adults: a population-based longitudinal study. Am J Epidemiol. 2017;186(9):1049-56.

Ready to submit your research? Choose BMC and benefit from:

- fast, convenient online submission

- thorough peer review by experienced researchers in your field

- rapid publication on acceptance

- support for research data, including large and complex data types

- gold Open Access which fosters wider collaboration and increased citations

- maximum visibility for your research: over $100 \mathrm{M}$ website views per year

At $\mathrm{BMC}$, research is always in progress.

Learn more biomedcentral.com/submissions 